\title{
Data Consistency in Emergency Management
}

\author{
D. Ergu, G. Kou, Y. Peng, F. Li, Y. Shi
}

\author{
Daji Ergu \\ College of Electrical and Information Engineering \\ Southwest University for Nationalities \\ Chengdu, China, 610041 \\ E-mail: ergudaji@163.com
}

\section{Gang Kou, Yi Peng, Feixiong Li}

School of Management and Economics

University of Electronic Science and Technology of China

Chengdu, China, 610054

E-mail: kougang@yahoo.com; pengyicd@gmail.com;

lifx@uestc.edu.cn

\section{Yong Shi}

1. Research Center on Fictitious Economy and Data Sciences

Chinese Academy of Sciences, Beijing 100190, China, and

2. College of Information Science \& Technology

University of Nebraska at Omaha, Omaha, NE 68182, USA

E-mail: yshi@gucas.ac.cn

\begin{abstract}
:
Timely response is extremely important in emergency management. However, cardinal inconsistent data may exist in a judgment matrix because of the limited expertise, preference conflict as well as the complexity nature of the decision problems. The existing inconsistent data processing models for positive reciprocal matrix either are complicated or dependent on the priority weights, which will delay the decision making process in emergency. In this paper, a geometric mean induced bias matrix (GMIBM), which is only based on the original matrix A, is proposed to quickly identify the most inconsistent data in the judgment matrix. The correctness and effectiveness of the proposed model are proved mathematically and illustrated by two numerical examples. The results show that the proposed model not only preserves most of the original information in matrix A, but also is faster than existing methods. Keywords: cardinal inconsistency, positive reciprocal matrix, geometric mean induced bias matrix (GMIBM), inconsistency identification
\end{abstract}

\section{Introduction}

Emergency management is an interdisciplinary field, and is in essence a complex multi-objective optimization problem [1]. Multi-criteria decision making (MCDM) methods have therefore been extensively employed to study emergency management, for instance, the Ordered Weighted Averaging (OWA) [2], Technique for Order Preference by Similarity to Ideal Solution (TOPSIS) [3], Preference Ranking Organization Method for Enrichment Evaluations (PROMETHEE) [4], Analytic Hierarchy Process (AHP) ( [5], [6], and [7]), Analytic Network Process (ANP) ( [8], [9]), Decision Making Trial and Evaluation Laboratory (DEMATEL) [10], Fusion Approach of MCDM (FAMCDM) methods [11] etc. Among these MCDM methods, AHP and ANP are two of the most popular methods for studying emergency management, and usually used to assess the emergency management performance, select the best emergency response alternatives or emergency recovery alternatives and allocate reasonable relief resources etc. 
In the AHP/ANP, the tangible and intangible attributes or criteria are always measured by numerical data through pairwise comparisons, and displayed in a judgment matrix whose numerical data are positive and reciprocal. The data of judgment matrix are usually provided by experts and collected through questionnaire survey [12]. Therefore, the data may be inconsistent because of the limited expertise, preference conflict as well as the complexity nature of the decision problems etc ( [13], [14]). For instance, assume there are three emergency response alternatives, $A, B$, and $C$, the $i^{\text {th }}$ expert thinks that alternative $A$ is preferred to $B 2$ times, and $B$ is preferred to $C 4$ times, but alternative $A$ is preferred to $C 3$ times in stead of 8 times. This is called cardinal inconsistency, and for an inconsistent judgment, the inconsistent data should be identified and adjusted before it is used to make a valid decision. Therefore, the inconsistent data processing issue in a judgment matrix has become a hot topic since the introduction of the AHP/ANP. Currently, there are many methods for identifying and adjusting the inconsistent data, for example, auto-adaptive algorithms in ( [14], [16]), absolute differences methods in ( [17], [18]), perturbation matrix method in [19] etc. However, the existing methods are either too complicated to delay the speed of inconsistent data analysis or are difficult to preserve most of the original information in the judgment matrix, or are extremely dependent on the priority weights. Therefore, it is necessary to propose a cardinal inconsistent data analysis model to effectively and simply identify the inconsistent data in order to make a valid decision, which is independent to the priority weights while preserving most of the original information in a judgment matrix. In an attempt to establish such models, the absolute differences of geometric mean matrix in [20], the induced bias matrix model (IBMM) in [21], were proposed to identify the possible inconsistent data in a judgment matrix.

In this paper, a geometric mean induced bias matrix (GMIBM), which is only based on the original matrix A, is established to identify the most inconsistent data in a judgment matrix. Through observing and adjusting the largest bias data in the induced bias matrix, the consistency ratio of the judgment matrix can be quickly improved to make a fast decision for emergence management. Besides, a general estimating formula of the mined cardinal inconsistent data of GMIBM is provided.

The remaining parts of this paper are organized as follows. The next section briefly describes the cardinal inconsistency in a judgment matrix. The theorems, corollaries and identifying processes of GMIBM are further proposed and presented in Section 3. Two numerical examples introduced in [21] are used to test the proposed model in Section 4. Section 5 concludes the paper.

\section{Cardinal Inconsistency}

Let the judgment matrix be $A=\left[a_{i j}\right]_{n \times n}$, where $a_{i j}>0$ and $a_{i j}=1 / a_{j i}$ for all $i, j$,and $k$, if $a_{i j}=a_{i k} a_{k j}$ holds for all $i, j$ and $k$, then matrix $A$ is said to be perfectly cardinal consistency, otherwise, it is called cardinal inconsistency. In practice, it is unrealistic to obtain a perfectly cardinal consistency of matrix $A$, therefore AHP allows a certain level of cardinal inconsistency of the judgment matrix. To measure the consistency of a judgment matrix and determine a certain acceptable level of inconsistency, Saaty proposed a consistency index (CI), denoted as:

$$
C I=\frac{\lambda_{\max }-n}{n-1}
$$

where $\lambda_{\max }$ is the maximum eigenvalue of matrix $A$, and $n$ is the order of matrix $A$.

To define a unique consistency test index that does not rely on the order of judgment matrices, the consistency index (CI) was extended and the consistency ratio (CR) method was further proposed by Saaty [17],

$$
C R=\frac{C I}{R I}
$$

where $C I$ is the consistency index shown in equation (1) while $R I$ is the average random index based on Matrix Size, as shown in Table 1. 
Table 1 The Average Random Index

\begin{tabular}{|l|l|l|l|l|l|l|l|l|l|l|}
\hline $\mathrm{n}$ & 1 & 2 & 3 & 4 & 5 & 6 & 7 & 8 & 9 & 10 \\
\hline $\mathrm{RI}$ & 0 & 0 & 0.52 & 0.89 & 1.11 & 1.25 & 1.35 & 1.4 & 1.45 & 1.49 \\
\hline
\end{tabular}

If the $C R$ of a judgment matrix is less than or equal to $0.1(C R \leq 0.1)$, indicating the inconsistency is relatively small, the judgment matrix is said to be of acceptable inconsistency. If $C R$ is greater than 0.1 $(C R>0.1)$, the judgment matrix is of unacceptable cardinal inconsistency, and the decision makers are asked to revise their judgments. To effectively identify the cardinal inconsistent data and improve the consistency ratio of a judgment matrix, a geometric mean induced bias matrix (GMIBM) by Hadarmad product is proposed in the following sections.

\section{Geometric Mean Induced Bias Matrix (GMIBM)}

\subsection{Theorem of GMIBM}

To identify the inconsistent data, a geometric mean induced bias matrix (for short GMIBM, hereinafter), which is only based on the original judgment matrix and independent to the priority weights, is established to amplify the most inconsistent data in this paper. Then, the most inconsistent data can be identified by observing the largest data in the induced bias matrix. The related theorems and corollaries are presented in this section.

Theorem 1. The geometric mean induced bias matrix (GMIBM) $C$ should be a $U$ matrix if judgment matrix A is perfectly consistent, that is,

$$
C=\bar{A} \circ A^{T}=\left(c_{i j}\right)=\left(\sqrt[n]{\prod_{k=1}^{n} a_{i k} a_{k j}} \cdot a_{j i}\right)=U \quad \text { if } \quad a_{i k} a_{k j}=a_{i j}
$$

where $\bar{A}=\left(\bar{a}_{i j}\right)_{n \times n}=\left(\sqrt[n]{\prod_{k=1}^{n} a_{i k} a_{k j}}\right)_{n \times n} \quad$ represents an $n$-by-n geometric mean matrix composed of all geometric mean of $a_{i k} a_{k j}$ for all $i, j$ and $k, U=\left[\begin{array}{ccc}1 & \cdots & 1 \\ \vdots & \ddots & \vdots \\ 1 & \cdots & 1\end{array}\right]$, n denotes the order of $A, A^{T}$ represents the transpose of matrix A. Symbol,$\circ /$ denotes Hadamard product (e.g. $C=A \circ B$ means $c_{i j}=a_{i j} b_{i j}$ for all $i$ and $j$ ).

Proof: If the judgment matrix satisfies the perfect consistency condition, namely, $a_{i k} a_{k j}=a_{i j}$ holds for all $i, j$ and $k$. Since $a_{i j}=1 / a_{j i}$, we have

$$
c_{i j}=\sqrt[n]{\prod_{k=1}^{n} a_{i k} a_{k j}} \cdot a_{j i}=\sqrt[n]{\prod_{k=1}^{n} a_{i j}} \cdot a_{j i}=\sqrt[n]{a_{i j}{ }^{n}} \cdot a_{j i}=a_{i j} a_{j i}=1
$$

Therefore, all entries in matrix $\mathrm{C}$ are ones if the matrix is perfectly consistent, and matrix $\mathrm{C}$ is a $U$ matrix, whose entries are ones.

To simply compute the GMIBM and easily understand the theorem of GMIBM, the Theorem 1 is transformed to following theorem. 
Theorem 2. The geometric mean induced bias matrix (GMIBM) $C$ should be a $U$ matrix if judgment matrix A is perfectly consistent, that is,

$$
C=L \times R \circ A^{T}=\left(c_{i j}\right)=\left(\sqrt[n]{\prod_{k=1}^{n} a_{i k}} \cdot \sqrt[n]{\prod_{k=1}^{n} a_{k j}} \cdot a_{j i}\right)=U \quad \text { if } \quad a_{i k} a_{k j}=a_{i j}
$$

where $L=\left(\sqrt[n]{\prod_{k=1}^{n} a_{i k}}\right)_{n \times 1}$ represents an n-by-one column matrix composed of geometric mean of rows in matrix $A$, while $R=\left(\sqrt[n]{\prod_{k=1}^{n} a_{k j}}\right)_{1 \times n}$ denotes an one-by-n row matrix composed of geometric mean of columns in matrix A.

Corollary 3. The geometric mean induced bias matrix (GMIBM) $C$ should be as close as possible to a $U$ matrix if judgment matrix $A$ is approximately consistent.

Corollary 4. There must be some inconsistent data in the geometric mean induced bias matrix (GMIBM) $C$ deviating far away from one if the judgment matrix is inconsistent.

Based on Corollary 4, we can identify the most inconsistent data in matrix $A$ by observing the largest data deviating from 1 in the geometric mean induced bias matrix (GMIBM) $C$. Details of inconsistency identification processes are presented below.

\subsection{Inconsistency Identification and Adjustment Processes of GMIBM}

To propose the inconsistency identification and adjustment processes of GMIBM based on above Theorems and Corollaries, the aforementioned $n$-by- $n$ judgment matrix $A=\left[a_{i j}\right]_{n \times n}$ is used in the following. The processes of inconsistent data analysis and adjustment of GMIBM include two major steps, inconsistency identification and inconsistency adjustment:

\section{Step I: Inconsistency Identification}

$\overline{\text { Step 1: Compute a column matrix } L}$ and a row matrix $R$, which are composed of geometric means of rows and columns respectively.

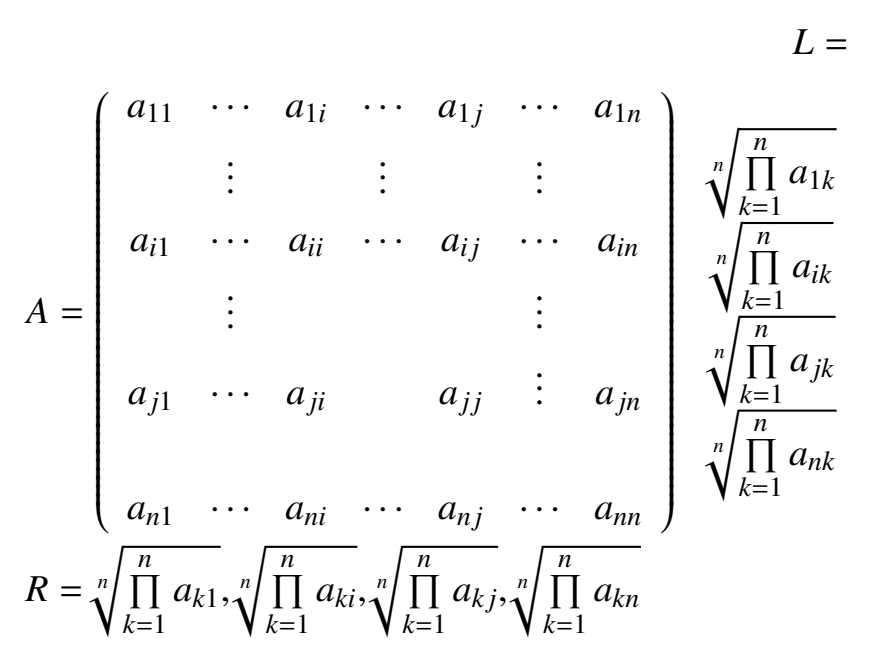

where

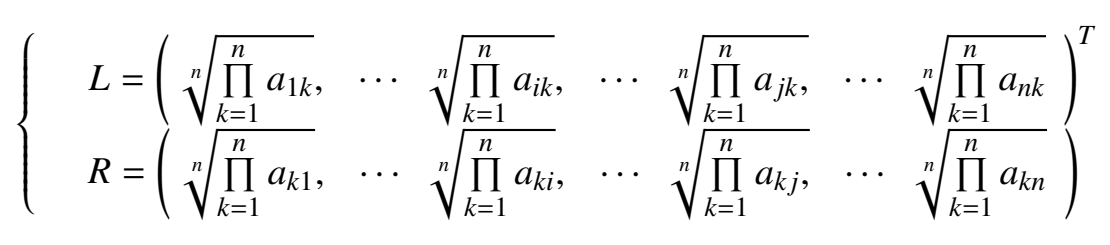


Step 2: Compute geometric mean matrix by formula

$$
\bar{A}=L \times R
$$

We can obtain the geometric mean matrix $\bar{A}$. The computing processes are shown below:

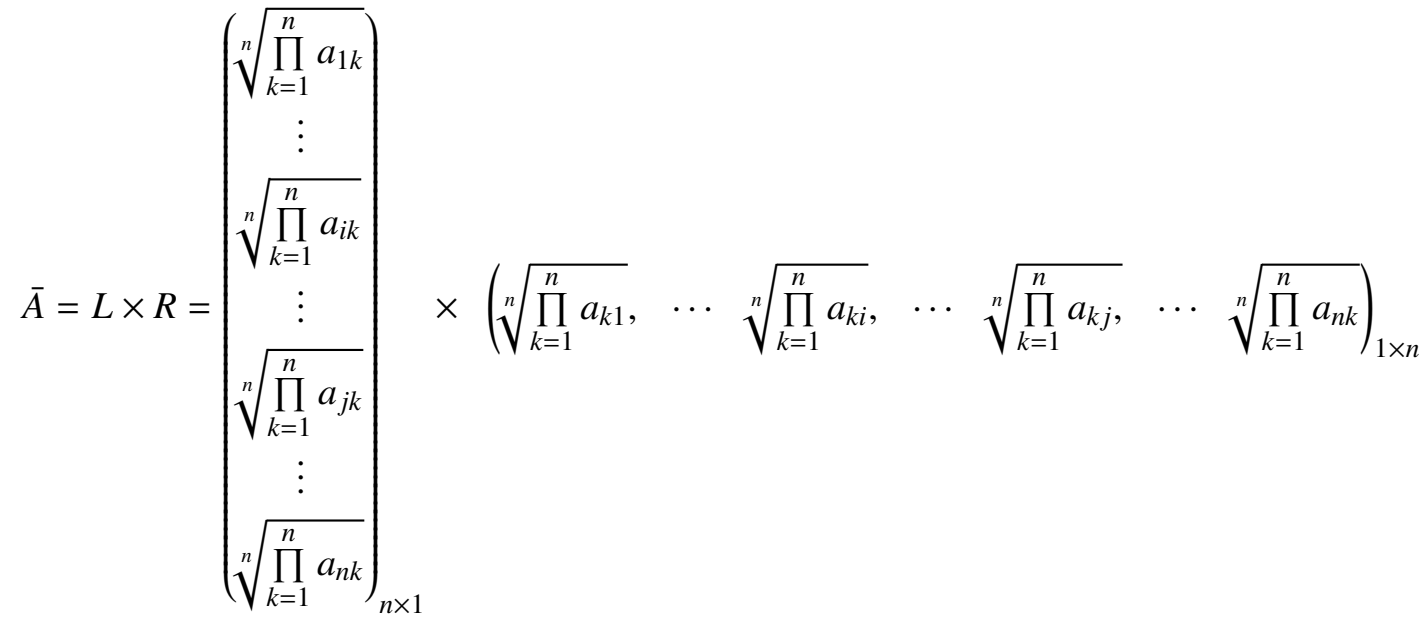

$$
\begin{aligned}
& =\left(\begin{array}{ccccccc}
\sqrt[n]{\prod_{k=1}^{n} a_{1 k} a_{k 1}} & \cdots & \sqrt[n]{\prod_{k=1}^{n} a_{1 k} a_{k i}} & \cdots & \sqrt[n]{\prod_{k=1}^{n} a_{1 k} a_{k j}} & \cdots & \sqrt[n]{\prod_{k=1}^{n} a_{1 k} a_{k n}} \\
\vdots & & \cdots & & \vdots & & \vdots \\
\sqrt[n]{\prod_{k=1}^{n} a_{i k} a_{k 1}} & \vdots & \sqrt[n]{\prod_{k=1}^{n} a_{i k} a_{k i}} & \cdots & \sqrt[n]{\prod_{k=1}^{n} a_{i k} a_{k j}} & \cdots & \sqrt[n]{\prod_{k=1}^{n} a_{i k} a_{k n}} \\
\vdots & & \vdots & & \vdots & & \vdots \\
\sqrt[n]{\sqrt[n]{\prod_{k=1}^{n} a_{j k} a_{k 1}}} & \cdots & \sqrt[n]{\prod_{k=1}^{n} a_{j k} a_{k i}} & \cdots & \sqrt[n]{\prod_{k=1}^{n} a_{j k} a_{k j}} & \cdots & \sqrt[n]{\prod_{k=1}^{n} a_{j k} a_{k n}} \\
\vdots & & \vdots & & \vdots & & \vdots \\
\sqrt[n]{\prod_{k=1}^{n} a_{n k} a_{k 1}} & \cdots & \sqrt[n]{\prod_{k=1}^{n} a_{n k} a_{k i}} & \cdots & \sqrt[n]{\prod_{k=1}^{n} a_{n k} a_{k j}} & \cdots & \sqrt[n]{\prod_{k=1}^{n} a_{n k} a_{k n}}
\end{array}\right)_{n \times n} \\
& =\left(\sqrt[n]{\prod_{k=1}^{n} a_{i k} a_{k j}}\right)_{n \times n}
\end{aligned}
$$

where $L$ is an $n$-by-one column matrix, which is composed of all geometric means of rows while $R$ is a one-by- $n$ row matrix $R$ composed of all geometric means of columns, as shown in formula (6) and the two edges of matrix A in Step 1 . The geometric mean matrix $\bar{A}$ can be easily computed by multiplying $L$ to $R$.

Step 3: Compute geometric mean induced bias matrix (GMIBM) $\mathrm{C}$ by formula,

$$
C=\bar{A} \circ A^{T}=\left(c_{i j}\right)=\left(\bar{a}_{i j} \cdot a_{j i}\right)=\left(\sqrt[n]{\prod_{k=1}^{n} a_{i k} a_{k j}} \cdot a_{j i}\right)
$$

Step 4: Identify the data with the largest value, denoted as $c_{i j}^{\max }$, deviating from 1 in matrix $\mathrm{C}$, then the corresponding $a_{i j}$ is regarded as the most inconsistent data in matrix $A$. If there are other data, say $c_{m n}, c_{p q}$, whose values are also deviating far away from 1 , then their corresponding data in matrix A, $a_{m n}, a_{p q}$, can also be considered as the possible inconsistent elements. Once the inconsistent data are 
identified, the following two steps are proposed to adjust the inconsistent data.

\section{Step II: Inconsistency Adjustment:}

Step 1: Estimate the value of identified inconsistent data by formula

$$
\tilde{a}_{i j}=\sqrt[n-2]{\prod_{k=1, \neq i, j}^{n} a_{i k} a_{k j}}=\sqrt[n-2]{\frac{\bar{a}_{i j}^{n}}{a_{i j}^{2}}}=\bar{a}_{i j}\left(\frac{\bar{a}_{i j}}{a_{i j}}\right)^{\frac{2}{n-2}}
$$

where $\tilde{a}_{i j}$ denotes the estimated value of the most inconsistent data $a_{i j}$ while $\bar{a}_{i j}$ is the geometric mean value located at the $i^{\text {th }}$ row and the $j^{\text {th }}$ column of geometric mean matrix $\bar{A}$.

Step 2: Test the consistency of the revised matrix A by replacing the inconsistent data with the estimated values.

To summarize, the processes of dealing with inconsistency by GMIBM include two major steps, that is, inconsistency identification and inconsistency adjustment. The first two steps in Step I are used to show the specific procedure of computing geometric mean matrix $\bar{A}$. For simplicity, one can directly use the latter two steps as the sub-steps of Step I to identify the most inconsistent data. To verify the effectiveness and accuracy of GMIBM, in the following, two numerical examples introduced in [20] are used to illustrate the proposed model.

\section{Illustrative Examples}

To test the effectiveness and correctness of the proposed GMIBM, and illustrate the processes of the proposed specific inconsistency identification and adjustment by numerical examples, two numerical examples in [21] are used in this paper.

Example 1 The Example 1 used in [21], which was firstly introduced in [20], is a $4 \times 4$ inconsistent pair-wise comparison matrix $A$ with $C R=0.173>0.1$.

$$
A=\left(\begin{array}{cccc}
1 & 1 / 9 & 3 & 1 / 5 \\
9 & 1 & 5 & 2 \\
1 / 3 & 1 / 5 & 1 & 1 / 2 \\
5 & 1 / 2 & 2 & 1
\end{array}\right)
$$

Apply the GMIBM to this matrix:

\section{Step I: Inconsistency Identification}

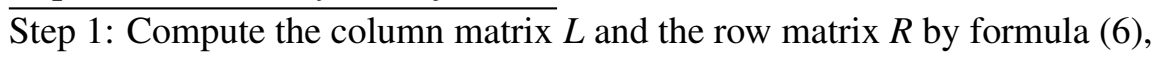

$$
L=\left(\begin{array}{llll}
0.5081 & 3.0801 & 0.4273 & 1.4953
\end{array}\right)^{T}, R=\left(\begin{array}{llll}
1.968 & 0.3247 & 2.3403 & 0.6687
\end{array}\right)
$$

Step 2: Compute geometric mean matrix by formula (7),

$$
\begin{aligned}
\bar{A} & =L \times R \\
& =\left(\begin{array}{cccc}
1 & 0.165 & 1.1892 & 0.3398 \\
6.0615 & 1 & 7.2084 & 2.0598 \\
0.8409 & 0.1387 & 1 & 0.2857 \\
2.9428 & 0.4855 & 3.4996 & 1
\end{array}\right)
\end{aligned}
$$


Step 3: Compute geometric mean induced bias matrix (GMIBM) C by formula (8),

$$
\begin{aligned}
C & =\bar{A}^{\circ} A^{T} \\
& =\left(\begin{array}{cccc}
1 & 1.4848 & 0.3964 & 1.6990 \\
0.6735 & 1 & 1.4417 & 1.0299 \\
2.5227 & 0.6936 & 1 & 0.5715 \\
0.5886 & 0.9710 & 1.7498 & 1
\end{array}\right)
\end{aligned}
$$

Step 4: Identify the largest value $c_{i j}^{\max }$ in matrix C. Here $c_{i j}^{\max }=c_{31}^{\max }=2.5227$, deviating from 1 in matrix $\mathrm{C}$, then the corresponding element $a_{31}$ in matrix $\mathrm{A}$ is regarded as the most inconsistent element,indicating that it is smaller than its average values.

\section{Step II: Inconsistency Adjustment}

Step 1: Estimate the possible proper value of $a_{31}$ using the estimating formula (9)

$$
\tilde{a}_{31}=\sqrt[4-2]{\frac{\bar{a}_{31}^{4}}{a_{31}^{2}}}=\sqrt{\frac{0.8409^{4}}{(1 / 3)^{2}}}=2.1213 \approx 2
$$

Step 2: Test the consistency of the revised matrix A by replacing the inconsistent elements $a_{31}$ and $a_{13}$ with the estimated values 2 and $1 / 2$. The revised matrix passed with $C R=0.0028<0.1$.

The identified inconsistent data and its estimated value are the same as the ones in [19] and [20], but the proposed method is faster to find the inconsistent element and estimate the values.

Example 2 The second example in [20] is a $4 \times 4$ inconsistent pair-wise comparison matrix $A$ with $C R=1.0242>0.1$.

$$
A=\left(\begin{array}{cccc}
1 & 2 & 4 & \frac{1}{8} \\
\frac{1}{2} & 1 & 2 & 4 \\
\frac{1}{4} & \frac{1}{2} & 1 & 2 \\
8 & \frac{1}{4} & \frac{1}{2} & 1
\end{array}\right)
$$

Apply the GMIBM to this matrix:

\section{Step I: Inconsistency Identification}

$\overline{\text { Step 1: Compute the column matrix }} R$ and the row matrix $L$ by formula (6),

$$
L=\left(\begin{array}{llll}
1 & 1.4142 & 0.7071 & 1
\end{array}\right)^{T}, \quad R=\left(\begin{array}{llll}
1 & 0.7071 & 1.4142 & 1
\end{array}\right)
$$

Step 2: Compute geometric mean matrix by formula (7),

$$
\begin{aligned}
\bar{A} & =L \times R \\
& =\left(\begin{array}{cccc}
1 & 0.7071 & 1.4142 & 1 \\
1.4142 & 1 & 2 & 1.4142 \\
0.7071 & 0.5 & 1 & 0.7071 \\
1 & 0.7071 & 1.4142 & 1
\end{array}\right)
\end{aligned}
$$


Step 3: Compute geometric mean induced bias matrix (GMIBM) C by formula (8),

$$
\begin{aligned}
C & =\bar{A}^{\circ} A^{T} \\
& =\left(\begin{array}{cccc}
1 & 0.3536 & 0.3536 & 8 \\
2.2828 & 1 & 1 & 0.3536 \\
2.2828 & 1 & 1 & 0.3536 \\
0.125 & 2.8284 & 2.8284 & 1
\end{array}\right)
\end{aligned}
$$

Step 4: Identify the largest value $c_{i j}^{\max }$ in matrix C. Here $c_{i j}^{\max }=c_{14}^{\max }=8$, deviating from 1 in matrix $\mathrm{C}$, then the corresponding element $a_{14}$ in matrix $A$ is regarded as the most inconsistent element, indicating that it is smaller than its average values.

\section{Step II: Inconsistency Adjustment}

Step 1: Estimate the possible proper value of $a_{14}$ using the estimating formula (9),

$$
\tilde{a}_{14}=\bar{a}_{14}\left(\frac{\bar{a}_{14}}{a_{14}}\right)^{\frac{2}{4-2}}=1 \cdot\left(\frac{1}{1 / 8}\right)=8
$$

Step 2: Test the consistency of the revised matrix A by replacing the inconsistent elements $a_{14}$ and $a_{41}$ with the estimated values 8 and $1 / 8$. The revised matrix passed with $C R=0<0.1$.

The identified inconsistent data and the estimated value are the same as the ones in [20], but the proposed method is faster to find the inconsistent data and estimate the values.

\section{Conclusions}

In this paper, we proposed a geometric mean induced bias matrix (GMIBM), which is only based on the original matrix and independent to the way of deriving the priority weights, to identify the cardinal inconsistent data in the judgment matrix. The inconsistent data identification process includes two major steps, namely, inconsistency identification and inconsistency adjustment. The inconsistent data can be easily and quickly identified by observing the data with the largest value(s) deviating from 1 in the induced bias matrix C. Besides, the identified data can be estimated by the estimated formula. Two examples are used to illustrate the proposed model. The results show that the proposed model is easier and faster to identify and adjust the inconsistent data than existing models.

\section{Acknowledgments}

This research has been partially supported by grants from Academic Degree Programs Construction at Southwest University for Nationalities (\#2012XWD-S1201), and grants from the National Natural Science Foundation of China (\#70901015 and \#70921061), the Fundamental Research Funds for the Central Universities and Program for New Century Excellent Talents in University (NCET-10-0293). No additional external funding received for this study. The funders had no role in study design, data collection and analysis, decision to publish, or preparation of the manuscript.

\section{Bibliography}

[1] Tufekci, S., Wallace, W.A, The emerging area of emergency management and engineering,IEEE Transactions on Engineering Management, 1998, 45(2):103-105, 1998.

[2] Shan, L., Zhao, Z.P, Evaluation on emergency management system in supply chain based on trapezoidal fuzzy order weighted average (FOWA) operator, Applied mechanics and materials,3435:1170-1174, 2010. 
[3] Chen, Y., Li, K., Xu, H.Y., Xu, H.Y, A DEA-TOPSIS method for multiple criteria decision analysis in emergency management,Journal of Systems Science and Systems Engineering, 18(4):489-507, 2009.

[4] Zhang, K., Kluck, C., Achari, G, A Comparative Approach for Ranking Contaminated Sites Based on the Risk Assessment Paradigm Using Fuzzy PROMETHEE, Environmental Management, 44(5):952967, 2009.

[5] Tie, Y.B. Tang, C, Zhou, C.H. The application of AHP to emergency response capability assessment in urban disaster, Journal of Geological Hazards and Environment Preservation, 16(4):433-437, 2005.

[6] Ergu, D., Kou, G., Peng, Y., Shi, Y., Shi, Y, The Analytic Hierarchy Process: Task Scheduling and Resource Allocation in Cloud Computing Environment,Journal of Supercomputing, DOI: 10.1007/s11227-011-0625-1, 2011.

[7] Wu, W.; Kou, G.; Peng, Y.; Ergu, D., Improved AHP-group decision making for investment strategy selection, Technological and Economic Development of Economy, 18(2), 2012. DOI: 10.3846/20294913.2012.680520

[8] Ergu, D., Kou, G., Shi, Y., Shi, Y., Analytic Network Process in Risk Assessment and Decision Analysis, Computers \& Operations Research, 2011. DOI: 10.1016/j.cor.2011.03.005.

[9] Levy J.K., Taji K., Group decision support for hazards planning and emergency management: A Group Analytic Network Process (GANP) approach, Mathematical and Computer Modelling,46(78): 906-917, 2007.

[10] Zhou Q., Huang W., Zhang Y., Identifying critical success factors in emergency management using a fuzzy DEMATEL method, Safety Science, 49(2):243-252, 2011.

[11] Peng, Y., Kou, G., Wang, G., and Shi, Y.,FAMCDM: A Fusion Approach of MCDM Methods to Rank Multiclass Classification Algorithms, Omega, 39(6): 677-689, 2011, DOI:10.1016/j.omega.2011.01.009

[12] Ergu, D., Kou, G., Questionnaire Design Improvement and Missing Item Scores Estimation for Rapid and Efficient Decision Making, Annals of Operations Research, DOI: 10.1007/s10479-0110922-3, 2011.

[13] Filip,F.G., D.A. Donciulescu, Cr.I. Filip.,Towards intelligent real-time Decision Support Systems for industrial milieu,Studies in Informatics and Control , 11 (4):303-311, 2001.

[14] Filip F.G., Decision support and control for large-scale complex systems, Annual Reviews in Control, 32(1):61-70,2008.

[15] Xu.Z, Wei.C ., A consistency improving method in the analytic hierarchy process, European Journal of Operational Research, 116:443-449, 1999.

[16] Cao, D., Leung, L.C., Law, J.S., Modifying inconsistent comparison matrix in analytic hierarchy process: A heuristic approach, Decision Support Systems ,44:944-953, 2008.

[17] Saaty, T.L., The Analytical Hierarchy Process, New York: McGraw-Hill, 1980.

[18] Saaty, T.L., How to Make a Decision: The Analytic Hierarchy Process, Interfaces, 24:19-43, 1994.

[19] Saaty, T.L., Decision-making with the AHP: Why is the principal eigenvector necessary, European Journal of Operational Research, 145(1): 85-91, 2003.

[20] Yang, Y.Q., Study on adjustment method for the inconsistency of the judgment matrix in AHP, Operations research and management science, 8(3):12-16,1999 (in Chinese).

[21] Ergu, D., Kou, G., Peng, Y., Shi, Y., A Simple Method to Improve the Consistency Ratio of the Pairwise Comparison Matrix in ANP, European Journal of Operational Research, 213(1):246-259, 2011. 